\title{
DIE NEUEN WAHRHEITSKRIEGE
}

\author{
von BERNHARD PÖRKSEN
}

Mit Donald Trump und Wladimir Putin regiere die Lüge, die Beliebigkeit der Postmoderne sei endgültig zum politischen Programm geworden, so heißt es. Aber stimmt das? Ein Essay über die Skandalisierung der Postmoderne. ${ }^{1}$

Es gibt ein kleines, fieses Theaterstück, das bereits in den Tagen nach der Wahl von Donald Trump von schockierten Intellektuellen und den Gegner_innen postmodernen Denkens rund um den Globus aufgeführt wurde. Dieses Theaterstück handelt von Schuld und Verfehlung in der Welt des Geistes. Es handelt davon, wie über den Umweg der postmodernen und konstruktivistischen Theorie-Mode das zersetzende Gift aus Lüge, Beliebigkeit und Spektakel allmählich zum politischen Programm geworden ist und den Horror des Populismus und der totalen Desinformation erst ermöglicht hat. «Indem Postmodernisten den wissenschaftlichen Objektivitätsanspruch unterminierten», so hieß es beispielsweise in typischer Manier im Scientific American, «haben sie unwissentlich die philosophische Grundlage für die Wiederkehr des Autoritarismus gelegt.» ${ }^{2}$ Silvio Berlusconi, die Kriegstreiber um George W. Bush, die mit der Lüge von Saddam Husseins Massenvernichtungswaffen den zweiten Irakkrieg rechtfertigten, der Autokrat Wladimir Putin, die Pro-Brexit-Trickser um Boris Johnson, der Reality-TV-Star und Präsident Donald Trump - sie alle werden derzeit als Profiteure des postmodernen und konstruktivistischen Relativismus gehandelt. Sie alle gelten als Nutznießer einer Denkweise, die davon ausgeht, dass wir im Erkennen alle Befangene sind, eingekapselt in soziale und biologische Prägungen, den Kokon aus Kultur und menschengemachter Perspektive, die das Erkennen absoluter Wahrheit unmöglich macht. Es ist eine Philosophie, die viele, sehr unterschiedliche Stichwortgeber_innen kennt. Zu ihnen zählen - je nach Perspektive - Jean Baudrillard oder Jean-François Lyotard, wahlweise auch Richard Rorty, Paul Feyerabend, Judith Butler oder Heinz von Foerster. Es ist eine Philosophie, die neben dem Abschied von der Wahrheit die Vielfalt der Lebensmöglichkeiten und die Akzeptanz kultureller Differenz als
1 Dieser Essay geht auf einen Text für die Wochenzeitung Die Zeit zurück, der neu bearbeitet und stark erweitert wurde: Bernhard Pörksen: Sind wir an alldem schuld?, in: Die Zeit, Nr. 6, 5.2.2017, 36. Siehe überdies ders.: Die große Gereiztheit. Wege aus der kollektiven Erregung, München 2018.

2 Shawn Otto: A Plan to Defend against the War on Science, in: Scientific American, dort datiert: 9.10.2016, scientificamerican.com/article/a-planto-defend-against-the-war-on-sciencel, gesehen am 25.10.2017. 
3 Vgl. Alan Sokal: Transgressing the Boundaries: Toward a Transformative Hermeneutics of Quantum Gravity, in: Social Text, Nr. 46/47, Spring/Summer 1996, 217-252.

4 Zur Rekonstruktion der SokalDebatte siehe das Abrechnungsbuch, das die Physiker Alan Sokal und Jean Bricmont verfasst haben: dies.: Eleganter Unsinn. Wie die Denker der Postmoderne die Wissenschaften missbrauchen, München 2001. Siehe überdies: Paul Boghossian: Sokals Jux und seine Lehren. Der postmoderne Schwindel - über den Niedergang wissenschaftlicher Standards und den Verlust intellektueller Verantwortung, in: Die Zeit, Nr. 5 , 24.1.1997, 49 f.

5 Barton Swaim: Clinton vs. Trump: Modern vs. postmodern, in: The Washington Post, dort datiert 27.9.2016, washingtonpost.com/blogs/ post-partisan/wp/2016/09/27/clintonus-trump-modern-us-postmodern/ ?utm_term=.4b86aoezaoeo, gesehen am 25.10.2017.

6 Michael Weiss: Donald Trump's Anti-Semitic Mob Came for Me, in: Daily Beast, dort datiert 21.6.2016, thedailybeast.com/donald-trumpsanti-semitic-mob-came-for-me, gesehen am 25.10.2017.

7 Felix Stephan: Mit den Waffen seiner Gegner, in: Zeit Online, dort datiert 10.11.2016, www.zeit.de| kultur/2016-11/donald-trumppraesident-usa-minderheiten-liberalitaet/, gesehen am 25.10.2017.

8 Peter Pomerantsev: Nothing is True and Everything is Possible.

The Surreal Heart of the New Russia, New York 2014, 33.

9 Boris Schumatsky: Der neue Untertan. Populismus, Postmoderne, Putin, Salzburg, Wien 2016, 99f., 104, 119. emanzipatorischen Aufbruch feiert, die statische Identitätskonzepte ablehnt, im Zweifel auch das scheinbar Natürliche zur sozialen Konstruktion und damit zur prinzipiell variablen Manövriermasse erklärt. Mitunter geraten ihre Vertreter_ innen - Stichwortgeber_innen der akademischen Welt in den I98oer und goer Jahren - auch in den Verdacht, selbst die Naturgesetze als bloße Erfindung zu begreifen, sich also endgültig ins Absurde zu verrennen. 1996 war dies auf spektakuläre Weise der Fall. Damals wurde die postmoderne Philosophie schon einmal vehement attackiert. Der New Yorker Physiker Alan Sokal war es, der sich einen Scherz mit globalen Schockeffekten erlaubte. Er schrieb im Jargon französischer Postmodernist_innen einen raffiniert gemachten Quatschessay, der letztlich auf die Schlussfolgerung zulief, die Naturgesetze seien die Erfindung toter, weißer Männer und im Dienste multikultureller Emanzipation grundsätzlich variabel. Sokals Nonsens-Text zur «transformativen Hermeneutik der Quantengravitation» wurde brav in einer berühmten kulturwissenschaftlichen Zeitschrift abgedruckt, was er selbst wenig später freudig enthüllte. ${ }^{3}$ Heute, gut 20 Jahre später, geht es härter und grundsätzlicher zur Sache. Der aktuelle Streit kennt keine Schlüsselfigur wie Alan Sokal, es gibt nicht nur den einen Anlass, den einen Fall. Aber es lässt sich eine allen Stellungnahmen gemeinsame These nachweisen, ein universales Prinzip der Skandalisierung. ${ }^{4}$ Diese These besagt, dass der politische Autoritarismus der Gegenwart auch das Resultat des intellektuell verwahrlosten Larifari-Denkens der Postmoderne sei.

Nun muss man kurz Luft holen und sich fragen: Ein solches Denken soll Donald Trump geprägt, Wladimir Putin beeinflusst, Silvio Berlusconi gestützt und die kriminellen Lügen der Kriegstreiber um George W. Bush begünstigt haben? Tatsächlich gilt Donald Trump als die «ironische, selbstreferentielle Verkörperung des postmodernen Wahrheitskonzeptes», ${ }^{\mathbf{5}}$ das aus den liberalen Philosophie-Departments in die Politik ausgewandert sei. Er sei der erste «postmoderne Herrscher der amerikanischen Geschichte». ${ }^{6}$ Sein Wahlsieg erscheint als der «finale Triumph der Postmoderne», ${ }^{7}$ der nur möglich gewesen sei, weil man den Begriff der Wahrheit ohnehin diskreditiert habe und man ihm daher seine zahllosen Lügen (bis zu 7 I Faktenverdrehungen in einer einstündigen Rede) nicht mehr wirklich krummnehmen konnte. Schriftsteller wie Peter Pomerantsev ${ }^{8}$ und Boris Schumatsky ${ }^{9}$ argumentieren ähnlich, aber sie nennen eine andere Angstfigur der Weltpolitik. Sie beschreiben in ihren aktuellen Essaybüchern Wladimir Putin als gelehrigen Schüler einer von nackten Machtinteressen geleiteten Simulationsmaschinerie. Sie porträtieren einen Mann, der sich bei all den Lügen über die Annexion der Krim, den Abschuss der MH 7 oder die Bombardierung von Aleppo an der freihändig übersetzten Nietzsche-Maxime eines entfesselten Konstruktivismus orientiert habe, ganz nach dem Motto: Es gibt keine Fakten, nur effektive Interpretationen! Der italienische Philosoph Maurizio Ferraris hatte hingegen schon vor Jahren den höchst einflussreichen Bush-Berater Karl Rove als philosophisch informierten Schurken im Visier («die USA sind jetzt ein Imperium, und wir schaffen uns 
unsere eigene Realität ...») und verdächtigt überdies Silvio Berlusconi als postmodernen Illusionskünstler. In seinem Manifest des neuen Realismus - gleichsam Auftakt und erster Akt des kleinen, fiesen Theaterstücks über die politischen Kollateralschäden des falschen Denkens - schreibt er 2012 den seither weltweit zitierten Satz: «Das, wovon die Postmodernen geträumt haben, haben die Populisten verwirklicht, und im Übergang vom Traum zur Wirklichkeit hat man erst wahrhaftig verstanden, worum es ging. $>^{\mathbf{1 0}}$

Tatsächlich sind die Belege für diese These, strikt empirisch gesprochen, äußerst schwach. Es sind immer dieselben fünf Beweis-Zitate eines verwahrlosten Denkens, die man zu lesen bekommt. Und niemand hat Donald Trump je bei der Lektüre des Philosophen Richard Rorty («Die Welt spricht nicht, nur wir sprechen») erwischt. Niemand hat ihn je über das wissenschaftstheoretische Motto von Paul Feyerabend («Anything goes!») oder Heinz von Foersters radikalen Konstruktivismus ( «die Umwelt, so wie wir sie wahrnehmen, ist unsere Erfindung») referieren hören. Kein Mensch hat Silvio Berlusconi je mit einem dieser kleinen, schlecht verleimten Merve-Bändchen herumstolzieren sehen, in denen Jean Baudrillard in dunkel schillernden Formulierungen die «Agonie des Realen» beschwört. Es ist auch sehr unwahrscheinlich, dass Wladimir Putin, nachdem er seine Soldat_innen 2014 auf der Krim einmarschieren ließ und ihre Präsenz erst offensiv dementierte, schließlich dann doch eingestehen würde, ein tatsächlich in jeder Hinsicht erbärmliches, peinlich-verspieltes Spiegel-Gespräch mit Jean Baudrillard aus dem Jahre I99 I gelesen zu haben, um sich strategisch in der Kunst der Realitätsleugnung zu schulen. Hier behauptet Baudrillard - auch dieses Gespräch wird gleichsam zu Tode zitiert -, der Golfkrieg sei eine Riesenshow und finde gar nicht wirklich statt." Das ist absurd, ganz klar. Hier redet jemand in vollendeter geistiger Provinzialität im System seines Jargons daher, der, selbst wenn Bomben fallen, bloß Simulation sehen kann. ${ }^{2}$ Nur: War dies je politisch wirksam? Und wen hat es eigentlich interessiert?

Man mag die aktuell aufflammenden Versuche einer Diffamierung postmoderner Philosophie durch bloße Assoziation für eine bizarre Blüte des Diskurses halten, gleichsam für eine sehr weltferne, allenfalls dürftig belegte Form des Streits. Aber die laufende Debatte ist eben doch (und dies gleich in mehrfacher Hinsicht) brisant. Zum einen stellen diejenigen, die sich hier zuschalten, die äußerst relevante und dem postmodernen Denken eng verwandte Frage, ob es eine Erkenntnistheorie des Widerstands geben könnte. Das ist der Impetus, der etwa einen scharfzüngig formulierenden Philosophen wie Markus Gabriel - auch er ein Protagonist des Neuen Realismus, Autor und Herausgeber zahlreicher Bücher zum Thema - umtreibt und ihn zu einem Erkenntnisprogramm in ethischmoralischer Absicht angeregt hat. ${ }^{13}$ Wie bricht man die Macht der Brutalo- und Nonsens-Narrative und reagiert auf Demagogie, Propaganda, Medienpopulismus? Was lässt sich - aus der Perspektive einer gesellschaftlich engagierten Wissenschaft - gegen die Schwächung des Arguments und die Umwertung der Werte tun? Welche <Philosophie> und welche Sprache, welche Formen und Instrumente
10 Maurizio Ferraris: Manifest des neuen Realismus, Frankfurt/M. 2014, 17.

11 Siehe Spiegel-Interview mit dem Pariser Kulturphilosophen Jean Baudrillard über die Wahrnehmbarkeit des Krieges: Claudius Seidl, Nikolaus von Festenberg: Der Feind ist verschwunden, in: Der Spiegel, Nr. 6, 1991, 220 f.

12 Zur Kritik siehe in aller gebotenen Schärfe: Susan Sontag: Das Leiden anderer betrachten, München, Wien 2003, 127.

13 Zum Programm des Neuen Realismus und der Sinnfeld-Ontologie siehe: Markus Gabriel: Warum es die Welt nicht gibt, Berlin 2013. Zu den Denkanlässen und Richtungen des Neuen Realismus liefern die einzelnen Aufsätze in folgendem Buch einen guten Überblick: Christoph Riedweg (Hg.): Nach der Postmoderne. Aktuelle Debatten zu Kunst, Philosophie und Gesellschaft, Basel 2014. 
14 Die Konstellationen, die einen Maurizio Ferraris inspiriert haben, werden in folgendem Beitrag charakterisiert: Peter Sloterdijk: Über Aktualität. Römische Fussnote zur Medientheorie, in: Riedweg ( $\mathrm{Hg}$.): Nach der Postmoderne, 291-298.

15 Zur Transformation heterodoxer Impulse in eine neue Orthodoxie (und der Kritik an der Erstarrung des Denkens) siehe z. B.: Heinz von Foerster, Bernhard Pörksen: Wahrheit ist die Erfindung eines Lügners. Gespräche für Skeptiker, Heidelberg 1998 , insbesondere $42 \mathrm{ff}$. Sowie: Humberto R. Maturana, Bernhard Pörksen: Vom Sein zum Tun. Die Ursprünge der Biologie des Erkennens, Heidelberg 2002, insbesondere $109 \mathrm{ff}$. der Intervention braucht es zu diesem Zweck? Das ist das eigentlich bedeutsame, unter flachen Provokationen verschüttete Anliegen, das einen Maurizio Ferraris und seine Anhänger_innen umtreibt, wenn sie für seinen Neuen Realismus und ein 〈starkes〉, an Tatsachen orientiertes Denken als 〈Gegenmacht $>$ zu einem alles zersetzenden Zweifel werben. ${ }^{14}$ Und tatsächlich, darüber lohnt es sich zu streiten. Zum anderen (und das macht die Kritik an der Postmoderne und das Werben um den Realismus in den Geistes- und Sozialwissenschaften zeitdiagnostisch brisant) zeigt sich hier eine Sehnsucht nach Verbindlichkeit, Orientierung und Gewissheit, die im Feld der geistes- und sozialwissenschaftlichen Theoriebildung Resonanz erzeugt. Auch dieser Appell, demokratische Werte und Grundrechte offensiv zu verteidigen, ist in Zeiten der allgemeinen Verunsicherung und des politischen Extremismus mehr als angebracht. Und schließlich: Das postmoderne Denken ist tatsächlich zu mächtig geworden - nur eben nicht im Weißen Haus, im Kreml oder in den Bling-Bling-Fernsehshows eines Berlusconi, sondern in den politisch eher einflusslosen Sinnprovinzen geistes- und sozialwissenschaftlicher Seminare. Hier ist die postmoderne Philosophie des antiautoritären Aufbruchs längst zur neuen Autorität mutiert. Hier liest man in den Einführungsbüchern für B.A.-Studierende der Geistes- und Sozialwissenschaften, dass eigentlich so ziemlich alles eine Konstruktion ist. Hier droht die Erstarrung des Denkens in Gestalt sektiererisch anmutender, kraftlos dahin gemurmelter, endlos wiederholter Glaubensbekenntnisse, die da heißen: «Es gibt keine Wahrheit»; «Objektivität ist ein Mythos»; «wir erfinden die Wirklichkeit». ${ }^{15}$

Ich habe, strikt anekdotisch und autobiografisch gesprochen, diese Verwandlung von antiautoritären Formeln in Mantren der Selbstvergewisserung ziemlich hautnah miterlebt. Vor knapp 20 Jahren schrieb ich mit dem Kybernetiker Heinz von Foerster, einem der Begründer des Konstruktivismus, mein erstes kleines Buch. Es trägt den Titel Wabrbeit ist die Erfindung eines Lügners. Foerster war, wie viele Protagonist_innen des Konstruktivismus und der Postmoderne, durchdrungen von dem Wunsch, das Denken gegen den Dogmatismus zu impfen. Er hatte die NS-Zeit als sogenannter Vierteljude in Berlin überlebt und schuf auf eine im Inneren erschütterte Weise eine Philosophie des fröhlichen Aufbruchs, eine heitere, elegante Anleitung zum Andersdenken, die ein ideologisches, gerade noch lebensgefährliches Wahrheitskonzept pulverisieren sollte. Sein Konstruktivismus war als Korrektiv gedacht, als eine Medizin gegen die Erstarrung, nicht als neues Glaubensbekenntnis für relativistische Sektierer_innen oder als Handreichung für Anhänger_innen und Epigon_innen, die in der Gründung von Schulen und der intellektuellen Konditionierung von Studierenden ihre Aufgabe sehen. Aber er wurde in hohem Alter zum Star der internationalen Wissenschaftsszene, gefeiert und bewundert. Und sah sich schließlich genötigt, die heillos Begeisterten vor den Gefahren eines konstruktivistischen Dogmatismus zu warnen, den sie, blind für den antiautoritären und antitotalitären Impuls, in ihrer Verehrung aus seinen Ideen schufen. Als ich ihm einmal vorschlug, sein Werk im Sinne eines konsequenten Skeptizismus durch den 
Versuch einer Demontage des Konstruktivismus zu krönen, musste er lachen. Er dachte kurz darüber nach, sah dann aber - im Gegensatz zu mir - sofort, dass einen ein solches Ansinnen unmittelbar wieder verbiesterungsanfällig werden lässt. Man ist nun in den semantischen Fangarmen des Gegners; man wird ihm ähnlich in der Geste der Aggression und gibt das Moment der Fraglichkeit und der ernsten Verspieltheit auf, von dem echtes, überraschungsfähiges Denken lebt. Nein, als konsequenter Konstruktivist, der im Antikonstruktivismus seine Lehre krönt, wollte er nicht verstanden werden. Eher ging es Heinz von Foerster darum, der Gefahr der vorschnellen Etikettierung zu entgehen, Erkenntnistheorie und Kommunikationspsychologie im Dienste wechselseitiger diskursiver Inspiration zu kombinieren. «Das, was Konstruktivismus genannt wird», so sagte er, sollte «schlicht eine skeptische Haltung bleiben, die die Selbstverständlichkeiten des Realismus in Zweifel zieht. Dann ließe sich vielleicht etwas freier sprechen. Man könnte auf andere Sichtweisen aufmerksam machen, sich von den schon vorgegebenen Urteilen und Denkweisen befreien. Das ist jedenfalls alles, was ich will. ${ }^{16}$ Und gerechnet hat mit der raschen Diffusion und Popularisierung der Konzepte (man denke nur an: das Prinzip der undifferenzierten Kodierung von Reizen aus der Außenwelt; das Lebenskriterium der Autopoiesis; die Idee der operativen Geschlossenheit von Systemen; die Entdeckung des Beobachters als zentrale Bezugsgröße allen Erkennens; die Operation des Unterscheidens als Fundamentaloperation des Denkens selbst) ohnehin keiner von denen, die heute als Urväter dieser Denkschule firmieren. ${ }^{17}$ Von Gregory Bateson bis zu Humberto Maturana, von Francisco Varela bis zu Ernst von Glasersfeld oder schließlich auch von Siegfried J. Schmidt bis hin zu Niklas Luhmann hat man den entstehenden Hype zunächst verwundert registriert, sich immer wieder jedoch auch von der drohenden Verflachung durch eine allzu rasche Popularisierung distanziert. ${ }^{18}$

Aber wie dem auch sei: Es stimmt gewiss, dass es in den Geistes- und Sozialwissenschaften tatsächlich eine erlebbare Diskursmacht der Postmoderne und des Konstruktivismus gibt, die allmählich zu Ende gehen könnte. Hier regiert ein fader, abgestanden wirkender Relativismus, wie der Soziologe Heinz Bude in einem seiner aktuellen Bücher, Das Gefübl der Welt, zu Recht moniert. «Auch das postmoderne Credo von der sozialen Konstruiertheit allen Wissens und aller Erkenntnis», so schreibt er hier,

und die daraus folgende Ethik der Anerkennung der vielen Arten und Weisen zu wissen und zu erkennen war ursprünglich ein großer Akt der Befreiung des Geistes von engstirnigen Methodologien und provinziellen Kosmologien. Wenn wir den konventionellen Status unserer Wissens- und Erkenntnisformen begreifen, so das Argument eines seinerzeit frischen und fröhlichen Denkens, dann können wir zu der wissenschaftspolitisch und alltagsmoralisch ungemein wichtigen Einsicht gelangen, dass wir selbst und nicht die Wirklichkeit dafür verantwortlich sind, dass wir wissen und erkennen. Aber auch diese Einsicht hat sich mit den Jahren von einer Behauptung der Öffnung in eine Doktrin der Schließung verwandelt. ${ }^{19}$
16 Von Foerster, Pörksen: Wahrheit ist die Erfindung eines Lügners, 45.

17 Zur Rezeption konstruktivistischer Konzepte in unterschiedlichen Disziplinen (Pädagogik, Managementwissenschaft, Sozialarbeit etc.) siehe: Bernhard Pörksen (Hg.): Schlüsselwerke des Konstruktivismus, Wiesbaden 2011.

18 Die Diffusion des Konstruktivismus aus der Neurobiologie und Kybernetik in die Literatur- und später die Medienwissenschaft wird von einer Fundamentalkritik begleitet, die an den methodologischen und methodischen Prämissen (man denke an die Arbeiten von Norbert Groeben) ansetzt. Ansonsten werden konstruktivistische Überlegungen intensiver vor allem von Medienwissenschaftlern wie etwa Florian Rötzer, Mike Sandbothe und Rudolf Maresch rezipiert, die sich mit postmoderner Philosophie bzw. Medientheorie insgesamt und den digitalen Medien auseinandersetzen. In Fachbüchern zur Kultur- und Medientheorie (wie z. B. in den Dubrovnik-Bänden von Hans Ulrich Gumbrecht und K. Ludwig Pfeiffer) übernimmt man dagegen die Überlegungen konstruktivistischer Wissenschaftler_innen in eher beschreibender, sachlicher Form als eine mögliche Perspektive unter vielen - ohne Kritik, ohne fachspezifische Ausdeutung.

19 Heinz Bude: Das Gefühl der Welt. Über die Macht von Stimmungen, München 2016, 27. 
Dieser Wechsel - von der Öffnung zur Schließung, vom Aufbruch zur Orthodoxie der Anti-Orthodoxie - lässt sich tatsächlich im universitären Milieu beobachten. Aber das heißt eben auch: Die gegenwärtig mit neuer Schärfe auftretenden Kritiker_innen von Postmoderne und Konstruktivismus verwechseln die Seminarsituation und den beherrschenden Denkstil sehr spezieller, vergleichsweise machtloser akademischer Milieus mit der allgemeinen politischen Großwetterlage. Ihre Pauschalkritik ist ein Lehrstück, das zeigt, wie man - nur scheinbar gegenwartsinteressiert - echte Probleme in akademische Hahnenkämpfe verwandelt und engagierte Zeitgenossenschaft bloß simuliert, strikt der fachinternen Taxonomie und einer gegenwartsfernen, interventionsuntauglichen Sprache und Hermetik verpflichtet. Überhaupt ist es genau diese Überschätzung der philosophischen Positionsstreitereien, die dem dringend notwendigen Engagement für die Verständigungsfähigkeit und die Wertebindung der Gesellschaft Energie raubt. Es würde gar nichts besser in der Welt, wenn man, wie der postmoderne Literaturtheoretiker Stanley Fish in einer hysterisch-entgleisten Gegenrede ${ }^{20}$ bemerkte, demnächst postmoderne Texte oder gar Autor_innen auf den Scheiterhaufen wirft oder konstruktivistische Denker_innen an der Einreise in die USA hindert. Und die Probleme der Gegenwart bestehen gewiss nicht darin, dass gerade irgendwo da draußen ein paar verrückte Baudrillard-Jünger_innen die Welt in Aufruhr versetzen. Das kann man nur glauben, wenn man taub geworden ist für die hasserfüllten Stimmen der Gegenwart, für die «Lügenpresse»-Schreie, die Attacken der Verschwörungstheoretiker_innen, die Verwünschungen, die die Trolle in den sozialen Netzwerken ausstoßen und die Ad-hoc-Einfälle eines amerikanischen Präsidenten, der Kriegsdrohungen gegenüber Syrien und Prahlereien mit Atomsprengköpfen gegenüber Nordkorea in Tweets verpackt. Das Problem ist ein in dieser Dimension neuartiger Propagandastil, eine Art des postmodernen Fundamentalismus, der die traditionellen akademischen Schemata zur Positionsbezeichnung durcheinanderwirbelt. Es handelt sich um eine bizarre Mischung aus Relativismus und brutaler Machtpolitik, aus totaler Skepsis und glashartem Dogmatismus. Und wenn man schon das gängige Ordnungsvokabular bemühen möchte: Donald Trump und Wladimir Putin verkörpern dieses erkenntnistheoretische Hybrid, eben die Zwitterform des postmodernen Fundamentalismus. Sie leugnen vom Klimawandel bis zum Bruch des Völkerrechts oder unangenehmen journalistischen Enthüllungen jede Realität, die ihnen nicht passt, und demontieren nach Belieben missliebige Gewissheiten in den endlosen Weiten der digitalen Öffentlichkeit. Sie unterhalten ihre eigenen Fake-News-Kanäle von der persönlichen Twitter-Präsenz bis hin zu Russia Today. Aber: Sie kennen, bei all ihrer Pseudoskepsis, eben eine einzige, ideologisch und nationalistisch eingefärb-

20 Vgl. Stanley Fish: Don't blame Nietzsche for Donald Trump, in: Foreign Policy, dort datiert 9.8.2016, foreignpolicy.com/2016/08/09/dontblame-nietzsche-for-donald-trumpl, gesehen am 25.10.2017. te Wahrheit, die sie fraglos durchsetzen wollen. Natürlich, es stimmt: Putin und Trump haben den Fundamentalzweifel in eine Waffe verwandelt, um Misstrauen zu schüren. Aber ist das die reine Lehre postmoderner Philosophie, ist das konsequent ausbuchstabierter Konstruktivismus? Sicher nicht, denn dieser Fundamentalzweifel gilt immer nur für die Ansichten der Gegner_innen, nie für die eigene 
Position. Sie sind Realisten im Blick auf eigene Positionen, Total-Skeptizisten im Blick auf missliebige, das eigene Weltbild irgendwie störende Ansichten. Wer diesen Propagandastil des instrumentalisierten Zweifels zur Dogmendurchsetzung dechiffrieren will, der sollte nicht irgendein Baudrillard-Büchlein lesen, sondern sich mit den jahrzehntelang eingesetzten Kampagnenmethoden der amerikanischen Tabakindustrie ( $\ll$ Rauchen ist unschädlich, Nikotin kein Suchtmittel») und den Verwirrungstechniken derjenigen befassen, die den menschengemachten Klimawandel leugnen. Hier stößt man auf ein düsteres PR-Skript, das tatsächlich politisch wirksam geworden ist und einem Dreischritt folgt. Schritt I: Man attackiere klassische Expert_innen und etablierte Institutionen der Wahrheitsermittlung. Schritt 2: Man baue selbst Pseudo-Expert_innen und scheinbar neutrale, medienkompetente Autoritäten und Organisationen auf, die mithelfen, empirische Gewissheit in bloße Meinungsartikel zu verwandeln. Schritt 3: Man feiere den manipulativ produzierten Zweifel öffentlich als Erfolg, um gleichzeitig unbeirrt an der Durchsetzung der eigenen Dogmen zu arbeiten.

Dieser Dreischritt ist gerade vor dem Hintergrund der aktuellen Kommunikations- und Medienbedingungen enorm erfolgreich, weil sich insgesamt eine publizistische Machtverschiebung zeigt, die Desinformation einflussreicher werden lässt. Zu einem systemischen Bild gehört, dass der etablierte Journalismus - einst zentrale Wahrheits- und Verifikationsinstanz - in den USA und Europa von einer Vertrauenskrise gebeutelt wird und die PR-Branche massiv expandiert. ${ }^{21}$ (Auf eine Journalistin oder einen Journalisten kommen in den USA inzwischen nahezu fünf PR-Macher_innen, die im Vergleich zu ihren journalistischen Kolleg_innen deutlich höhere Gehälter beziehen). Hinzu kommt, dass Digitalgiganten den Werbemarkt kannibalisieren (von einem Werbedollar gehen durchschnittlich 6o Cent an Google oder Facebook) und auf diese Weise die ohnehin gegebenen Refinanzierungsprobleme der Qualitätspublizistik verschärfen. Allerdings: Man kann im Sinne der Medium-Theorie (Joshua Meyrowitz) die erlebbare Evidenzkrise auch - aus einer umfassenderen Perspektive - als Ergebnis einer «Deregulierung des Wahrheitsmarktes» ${ }^{22}$ begreifen, die Kontingenzerfahrungen durch die radikale Pluralisierung der Perspektiven intensiviert. Wer mag, kann sich barrierefrei zuschalten und seine Meinungen und Ansichten in die Erregungskreisläufe einspeisen. ${ }^{23}$ Bereits die Art der Informationspräsentation in sozialen Netzwerken und digitalen Öffentlichkeiten suggeriert im Übrigen eine epistemische Gleichwertigkeit, die so nicht existiert, denn es fehlen in der Regel definierende Glaubwürdigkeitssignale - im Extremfall steht die Lüge unmittelbar neben der Wahrheit, der Unsinn in direkter Konkurrenz zum Sinn. Man könnte sagen: Es regiert hier eine wortlose Ideologie, die ich die Gleichwertigkeitsdoktrin in der Informationspräsentation nennen möchte. ${ }^{24}$ Auch auf dem Smartphone, in der eigenen Timeline fließen Informationen sehr unterschiedlicher Qualität und Provenienz relativ unterschiedslos zusammen - ganz so, als handele es sich um völlig zu Recht unmittelbar rivalisierende Wirklichkeiten. Und schließlich und endlich gilt grundsätzlich: Einmal digitalisierte Information ist beweglich, hoch
21 Zum Folgenden siehe auch Bernhard Pörksen: Die Deregulierung des Wahrheitsmarktes. Von der Macht der Desinformation im digitalen Zeitalter, in: Günter Blamberger, Axel Freimuth, Peter Strohschneider (Hg.): Vom Umgang mit Fakten. Antworten aus Natur-, Sozial- und Geisteswissenschaften, Paderborn 2018 (im Erscheinen).

22 Die Formulierung von der Deregulierung des Wahrheitsmarktes entnehme ich einem Essay von Michael Seemann: Digitaler Tribalismus und Fake News, in: ctrl-verlust. net, dort datiert 29.9.2017, ctrlverlust.net/digitaler-tribalismus-undfake-news/, gesehen am 10.10.2017.

23 Vgl. Jayson Harsin: Regimes of Posttruth, Postpolitics, and Attention Economies, in: Communication, Culture \& Critique, Vol. 8, Nr. 2., 2015, 327-333.

24 Zum Begriff der Gleichwertigkeitsdoktrin siehe ursprünglich: Paul Boghossian: Angst vor der Wahrheit. Ein Plädoyer gegen Relativismus und Konstruktivismus, Berlin 2013, 10. 
25 Vgl. hierzu die Arbeiten von Joshua Meyrowitz und Neil Postman, siehe überdies Pörksen: Die große Gereiztheit, insbesondere 24-61. Desweiteren sei - im Sinne eines Hintergrundbildes und einer grundsätzlichen Positionierung, die ich teile - auffolgenden Beitrag verwiesen: Melvin Kranzberg: Presidential Address. Technology and History: "Kranzberg's Laws", in: Technology and Culture, Vol. 27, Nr. 3, 544-560. 26 Zu der allmählichen Verwandlung der Autorenexistenz des universitären Intellektuellen in die Indikatorenexistenz des Forschungsmanagers unter den Bedingungen der gegenwärtigen Wissenschaftspolitik siehe die vom Autor eröffnete, über mehrere Monate hinweg andauernde Zeit-Debatte, die sich unter folgendem Link findet: $w w w$. zeit.de/serie/wo-seid-ihr-professoren, gesehen am 8.7.2018. reaktiv, kann leicht von Plattform zu Plattform und von Netzwerk zu Netzwerk diffundieren. Im Kopieren, im Verlinken und wechselseitigen Zitieren werden so womöglich auch komplett erfundene Behauptungen aufgewertet (man denke nur an \#pizzagate) - und gelangen von den Rändern und den Schmuddelecken des Diskurses in einem Prozess der Informationswäsche und der Quellenverdunkelung sehr direkt und sehr rasch ins Zentrum der öffentlichen Welt. Das heißt, allgemeiner betrachtet, dass sich die Vermittlung von Informationen im flüssigen, beweglichen Aggregratzustand des Digitalen auf eine andere Ebene verlagert, sich gleichsam in die Horizontale hinein bewegt, ohne eine präfabrizierte, vergleichsweise stabile Unterscheidung von Peripherie und Zentrum, die noch in einer von Printmedien bestimmten Kultur sehr viel leichter stabilisierbar war. ${ }^{25}$

Was folgt aus all dem im Sinne einer verallgemeinernden Bilanz? Deutlich wird, dass es an der Zeit ist, die aktuellen Science Wars und die Skandalisierung der Postmoderne als eine Form der Selbstblockade und der Selbstentfremdung von echten Problemen zu begreifen. Eine solche Debatte geht zu Lasten einer wachen, interventionsbereiten Ideologieanalyse. Man sieht, wie sich das Bemühen um Relevanz letztlich in die weltabgewandte Irrelevanz von rein innerakademischen Positionskämpfen verwandelt hat. Was es jetzt braucht, ist ein Abschied vom Schulen- und Schurkendenken, um jenseits der spektakulär inszenierten Paradigmenkämpfe für den Wert einer offenen Gesellschaft und die Bedeutung empirisch gesicherten Wissens zu streiten. Es gilt gegen einen autoritären, bizarren Irrationalismus anzugehen, der Wahrheitsfuror und Beliebigkeitsdenken eigentümlich vermischt und der hier als postmoderner Fundamentalismus bezeichnet wurde. Das wäre eine gemeinsame Vision, eine echte Aufgabe, die die Geistes- und Sozialwissenschaften wieder näher an die Gegenwart und die Dramen der wirklichen Welt heranführen könnte. Sie müssten allerdings, damit dies überhaupt gelingen kann, die Kunst der Einmischung und der öffentlichen Intervention nach einer Phase der systematischen Entwertung und Marginalisierung von Debattenbüchern, Polemiken und reaktionsschnell formulierten Essays erst wieder lernen. Sie müssten sich von fachintern äußerst wirkmächtigen, seltsam einschüchternd wirkenden Unterscheidungen (Prominenz versus Reputation, Oberfläche versus Tiefe, Fachlichkeit versus Feuilletonismus) lösen bzw. diese zumindest auch als Diskursblockaden begreifen. Sie müssten sich bei der Wahl der sprachlichen Mittel von der Tabuisierung der Zuspitzung verabschieden, die heute in vielen Disziplinen implizit verlangt wird - und manchmal auch ganz explizit als Ausweis von Wissenschaftlichkeit gilt. ${ }^{26}$ Sie müssten, mit einem Wort, überhaupt öffentlich wirken wollen. All dies wäre nötig, denn was sich heute beobachten lässt, ist kein Streit philosophischer Schulen, keine im Scholastischen beheimatete Debatte. Man mag dies bedauern oder ignorieren, aber die Wahrheitskriege der neuen Zeit finden nicht mehr im Seminarraum statt. 